\title{
Transversal Stiffness and Beta-Actin and Alpha-Actinin-4 Content of the M. Soleus Fibers in the Conditions of a 3-Day Reloading after 14-Day Gravitational Unloading
}

\author{
I. V. Ogneva \\ State Scientific Center of Russian Federation-Institute of Biomedical Problems, Russian Academy of Sciences, 76-a, \\ Khoroshevskoye shosse, Moscow 123 007, Russia \\ Correspondence should be addressed to I. V. Ogneva, iogneva@yandex.ru
}

Received 2 April 2011; Revised 7 July 2011; Accepted 22 July 2011

Academic Editor: J.-P. Jin

Copyright (๑) 2011 I. V. Ogneva. This is an open access article distributed under the Creative Commons Attribution License, which permits unrestricted use, distribution, and reproduction in any medium, provided the original work is properly cited.

The aim of the work was to analyze the structural changes in different parts of the sarcolemma and contractile apparatus of muscle fibers by measuring their transversal stiffness by atomic force microscopy in a three-day reloading after a 14-day gravity disuse, which was carried out by hind-limbs suspension. The object of the study was the soleus muscle of the Wistar rat. It was shown that after 14 days of disuse, there was a reduction of transversal stiffness of all points of the sarcolemma and contractile apparatus. Readaptation for 3 days leads to complete recovery of the values of the transversal stiffness of the sarcolemma and to partial value recovery of the contractile apparatus. The changes in transversal stiffness of sarcolemma correlate with beta-actin and alpha-actinin-4 in membrane protein fractions.

\section{Introduction}

Results of a large number of studies in terms of real and simulated gravitational unloading suggest that in different organs and tissues negative changes are formed as a result of microgravity. Skeletal muscle is particularly exposed to weightlessness as a specialized organ that performs postural and motor functions. The subject of many studies is the soleus muscle, for which it was shown that prolonged exposure to microgravity conditions leads to a significant reduction of its mass, atrophic changes of the fibers [1-3]. Under the conditions of gravitational unloading, synthesis of myosin heavy chain of fast type is increased in the muscle fibers [3-6]. Atrophic changes and reduction of the number of slow fibers lead to a rapid transformation [7]. As a result, contraction time decreases, and soleus muscle becomes less resistant to prolonged stress $[8,9]$, and there is a decrease in functionality both of the whole muscle $[10,11]$ and single fibers [12].

But equally significant problem facing the human exploration of outer space, including missions to Mars, is the early period of readaptation to gravity. Particularly important is to restore the functionality of the muscles, the acceleration of which is not possible without understanding the mechanism of readaptation changes.

It was shown that the readaptation to gravity of atrophied muscles leads to more pronounced negative changes than in microgravity. It became clear after SLS-2 mission (1993), during which tissues were taken in conditions of weightlessness. Serious damage to the fibers of $m$. soleus and $m$. adductor longus in these samples was absent, as well as after 3 hours of readaptation, and became prominent after 4.5 hours of stay [13]. Analyzing the results of this experiment, Riley et al. [13] concluded that the contractile activity in the early period of readaptation of atrophied muscle is perceived as an eccentric load, as sarcomere structure damages, observed after 4.5 hours of reloading, are similar to those after eccentric contractions [14-17].

After 8-11 hours in conditions of Earth's gravity after a 14-day space flight of the biosatellite "Kosmos 2044," Riley et al. [14] showed that there were extensive damages to the sarcomere of $m$. adductor longus fibers, minimal necrosis in the tissue, and a slight activation of macrophages 
and satellite cells. However, a 2-day reloading after 12.5day stay in weightless conditions on board the biosatellite "Kosmos 1887" leads to more extensive necrosis, a significant activation of macrophages and satellite cells [15].

At the same time, after 3 days of reloading after a 10day antiorthostatic weighing of the rats, the levels of phosphorylated $\mathrm{PKB} / \mathrm{Akt}$ and $\mathrm{p} 70 \mathrm{~S} 6 \mathrm{~K}$ were significantly increased (by $56 \%$ and $26 \%$, resp.) in relation to control, to which they returned after 10 days of readaptation [18]. This may indicate an intensification of protein synthesis in muscle fibers during the period of 3-10 days of readaptation to gravity in $1 \mathrm{G}$. Perhaps due to the intensification of protein synthesis after 7 days of reloading after 7- and 14-day antiorthostatic weighing of the rats, an increase in cross-sectional area of soleus fibers to the level of control was observed [19].

After 14 days of readaptation after 21-day weighing of the rat, the maximum force of contraction of the soleus muscle was increased compared to that immediately after gravitational unloading [11], whereas after 9 days of stay in $1 \mathrm{G}$ after a 15-day antiorthostatic weighing of the rat, it was not improved [10]. However, according to Litvinova et al. [20], the maximum force of contraction of single fibers of the soleus muscle after 3- and 7-day reloading after a 14-day gravity discharge did not differ from the level immediately after the weighing. Nonetheless, after 14 days of readaptation after a 21-day discharge, almost complete restoration of the structure of the sarcomere was observed [11].

At the same time, the muscle fiber is a three-dimensional structure in which the tension transmission occurs not only longitudinally, but also in the transverse direction [21]. Changes in the structure of muscle fibers, observed in a gravity disuse and follow-up readaptation period, naturally lead to a change in mechanical properties that depend on the integrity of the structure, such as stiffness. In turn, changes in the longitudinal and transversal stiffness are directly related to the changes in contractile properties of muscle fibers $[12,22,23]$. The results of the study of transversal stiffness of contractile apparatus suggest that gravitational unloading caused different changes in slow and fast muscles. It was shown that hindlimb suspension led to decrease of transversal stiffness of soleus fibers and increase of transversal stiffness of tibialis anterior fibers [23]. At the same time, visual observations of the structure of individual compartments of muscle fibers, such as sarcolemma, are virtually impossible, and to assess its structural changes, transversal stiffness may be useful. Structural basis of the transversal stiffness of the sarcolemma with cortical cytoskeleton is a network of filaments formed by nonmuscle actin-beta-actin. To form such a network, a host of capping proteins is needed, one of which is alpha-actinin-4, attention to which in a gravity disuse is due primarily to the fact that its bind-ing to beta-actin depends on the content of calcium ions, the increase of which in these conditions has been shown previously $[22,24,25]$.

Thus, in spite of some data on the dynamics of reloading of the various parameters characterizing the structural and functional properties of the soleus muscle, the changes at the cellular level have hardly been studied. However, the implementation of readaptation process, as a special case of the reaction to changes in external mechanical conditions, at the cellular level is a key element in restoring the health of the muscles after a long stay in weightlessness.

Previously proposed method [26] of determination of the structure condition, based on measuring the mechanical characteristics of the different compartments of muscle fibers by atomic force microscopy, can assist in assessing the changes being implemented not only at the level of the contractile apparatus, but also at the level of the sarcolemma in readaptation to gravity after being in microgravity conditions.

\section{Materials and Methods}

Experiments were performed on the soleus muscle fibers (Sol) of a Wistar rat weighing 225-255 g. In order to simulate the conditions of gravitational unloading in rodents, antiorthostatic weighing of their hind limbs was conducted by the method of Ilyin-Novikov in the modification by MoreyHolton [27]. The duration of gravitational unloading in rats was 14 days. Reloading within 3 days was performed in the same conditions as Vivary control. The choice of this period of recovery was due, on the one hand, to the data [15] on even more significant damage to the sarcomere compared with the state immediately after unloading, and on the other hand, the data [18] on the beginning of the intensification of protein synthesis. Thus, reloading within 3 days after 14day gravity disuse is of fundamental importance for understanding the cellular mechanisms of the acute period of readaptation. The following groups were formed: "Control," "14-HS," and "14-HS + 3-R" of 7 animals each.

All procedures with animals were approved by the Commission on Biomedical Ethics SSC RF Institute for Biomedical Problems, RAS.

2.1. Atomic Force Microscopy. To determine the transversal stiffness of the different compartments of muscle fibers, atomic force microscopy was used. The method of obtaining preparations for research and images of the surface of muscle fibers that allow for local measurements of the transversal stiffness we described in detail earlier $[23,26]$.

The muscle was excised from tendon to tendon and, in order to carry out a partial destruction of the cell membrane, it was treated according to the method of chemical skinning, which was previously described by Stevens et al. [28]. Before the time of the experiments, the samples were stored at $-20^{\circ} \mathrm{C}$ in a buffer-containing relaxing solution in equal parts by volume of $\mathrm{R}$ ( $20 \mathrm{mM}$ MOPS, $170 \mathrm{mM}$ potassium propionate, and $2.5 \mathrm{mM}$ magnesium acetate, $5 \mathrm{mM} \mathrm{K}_{2}$ EGTA, $2.5 \mathrm{mM}$ ATP) and glycerol.

On the day of the experiment, the samples were transferred into solution $\mathrm{R}$, in which single-permeabilized muscle fibers were isolated.

To obtain demembranated fibers, single-permeabilized muscle fibers, which were in solution $\mathrm{R}$, were incubated with detergent Triton X-100, the final concentration of which was $2 \%$ by volume for 12 hours at a temperature of $+4^{\circ} \mathrm{C}$. After 
treatment with detergent, the obtained fibers were washed in the solution R.

To measure the transversal stiffness, the isolated fibers were attached to the bottom of the liquid cell of atomic force microscope, fixing their ends with a special glue Fluka shellac wax-free (Sigma, Germany). Depending on the series of experiments, a cell was filled either with a relaxing solution $\mathrm{R}$ or an activating solution A (20 mM MOPS, $172 \mathrm{mM}$ potassium propionate, $2.38 \mathrm{mM}$ magnesium acetate, $5 \mathrm{mM}$ CaEGTA, and $2.5 \mathrm{mM}$ ATP), or rigor solution $\mathrm{Rg}(20 \mathrm{mM}$ MOPS, $170 \mathrm{mM}$ potassium propionate, $2.5 \mathrm{mM}$ magnesium acetate, and $5 \mathrm{mM} \mathrm{K} \mathrm{K}_{2} \mathrm{EGTA}$ ).

The measurements of transversal stiffness of both permeabilized and Triton-treated fibers were performed using the platform Solver-P47-Pro (NT-MDT, Russia). A preliminary scan of the surface allowed determining the length of the sarcomere as described previously [26], which was $2.48 \pm$ $0.03 \mu \mathrm{m}$. The indentation depth was $150 \mathrm{~nm}$. To obtain each average value, at least 35 measurements were conducted.

Processing of the results was carried out in a specially designed program in MatLab 6.5 environment.

2.2. Western Blotting. To determine protein content, the muscle was frozen at liquid nitrogen temperatures. Preparation of tissue extracts and generation membrane and cytoplasmic fraction were carried out by the method of Vitorino et al. [29]. Denaturing polyacrylamide gel electrophoresis was carried out by the method of Laemmli on Bio-Rad system (USA), as previously mentioned in [23]. Based on the measurements of concentration, each well was applied the same amount of protein. Transfer to nitrocellulose membrane was carried out by the method of Towbin et al. [30].

In order to determine each protein, the specific primary monoclonal antibodies based on mouse immunoglobulin (Sigma, Germany) were used in the dilutions recommended by the manufacturer: for beta-actin-in a dilution of $1: 500$, for alpha-actinin-4-1:400. As secondary antibodies, biotinylated goat antibodies were used against mice IgG (Sigma, Germany) in a dilution of $1: 6000$. Next, the membranes were treated with a solution of streptavidin conjugated with horseradish peroxidase (Sigma, Germany) in a dilution of $1: 10000$. Protein bands were detected using $3,3^{\prime}$-diaminobenzidine (Merck, USA).

2.3. Statistical Analysis. The results obtained in the experiments results were statistically processed using ANOVA, using for the validation of the differences between the groups post hoc $t$-test with a significance level of $P<0.05$. The data were presented as $\mathrm{M} \pm \mathrm{SE}$, where $\mathrm{M}$ is the mean value of the estimated values, and SE is the standard error of mean value.

\section{Results}

3.1. Transversal Stiffness of Various Regions of the Contractile Apparatus and the Sarcolemma of Sol Single Fibers. In the "Control" group, the transversal stiffness of Tritontreated fibers (Figure 1(a)) in semisarcomere area, that is, between the Z-disk and M-line, significantly increases from

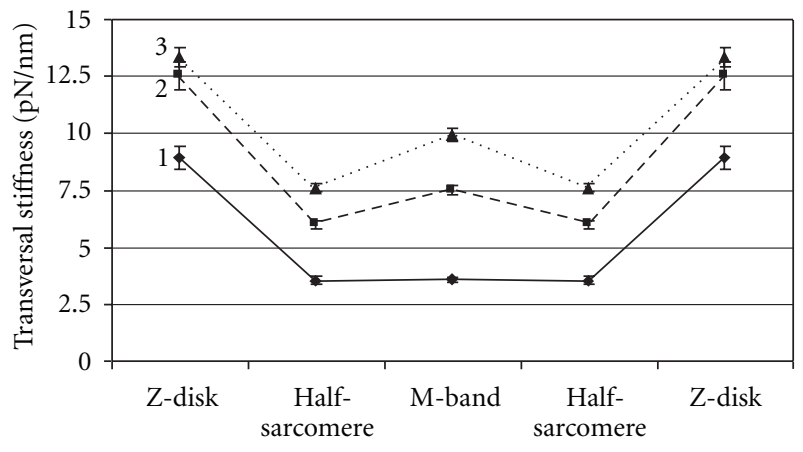

(a)

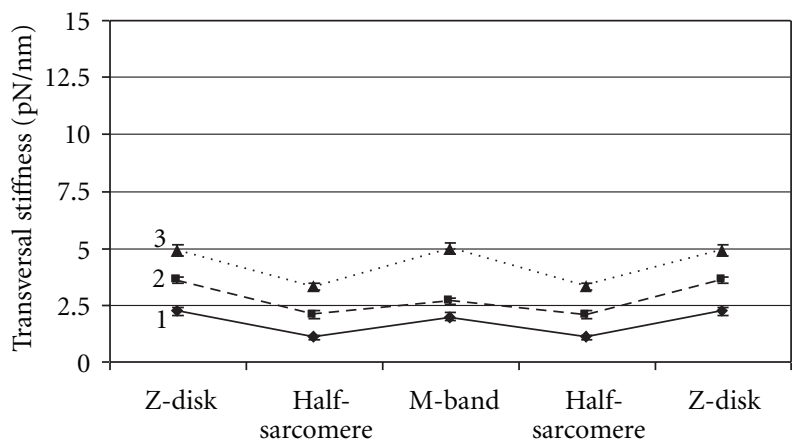

(b)

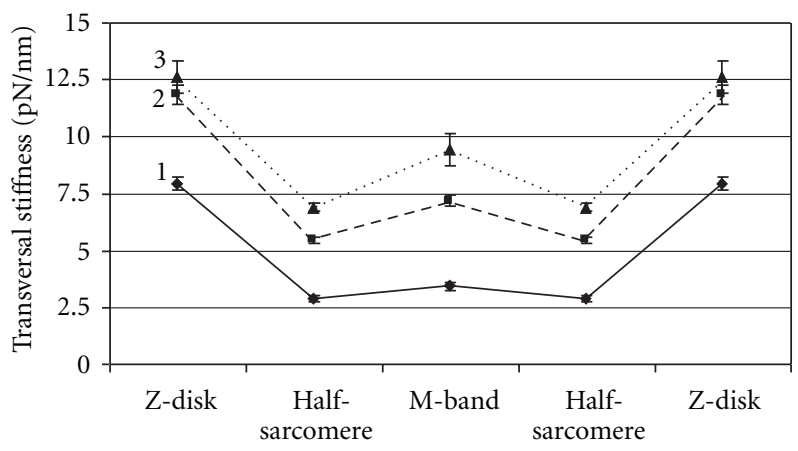

(c)

FIGURE 1: Regional distribution of the transversal stiffness along the sarcomere of isolated Triton-treated Sol muscle fibers in liquid in relaxed (1), calcium activated (2), and rigor (3) states after 14-day gravitational unloading and 3-day reloading; A: group "Control," B: group “14-HS," C: group “14-HS + 3-R.”

the relaxed state to the activated and from activated to rigor. After a 14-day gravitational unloading, transversal stiffness of all Triton-treated fibers (Figure 1(b)) is significantly reduced, relative to similar measurement points in the "Control" group, especially in the Z-disk area; however, a significant increase in stiffness, typical of all sections of the fibers at contraction activation and transition to the rigor, is preserved. After a 3-day reloading (Figure 1(c)), values of the transversal stiffness of Triton-treated fibers in semisarcomere area in all the states are still significantly below the level of control, but higher than those in the "14-HS" group. In addition, the transversal stiffness of Z-disk of fibers in the 

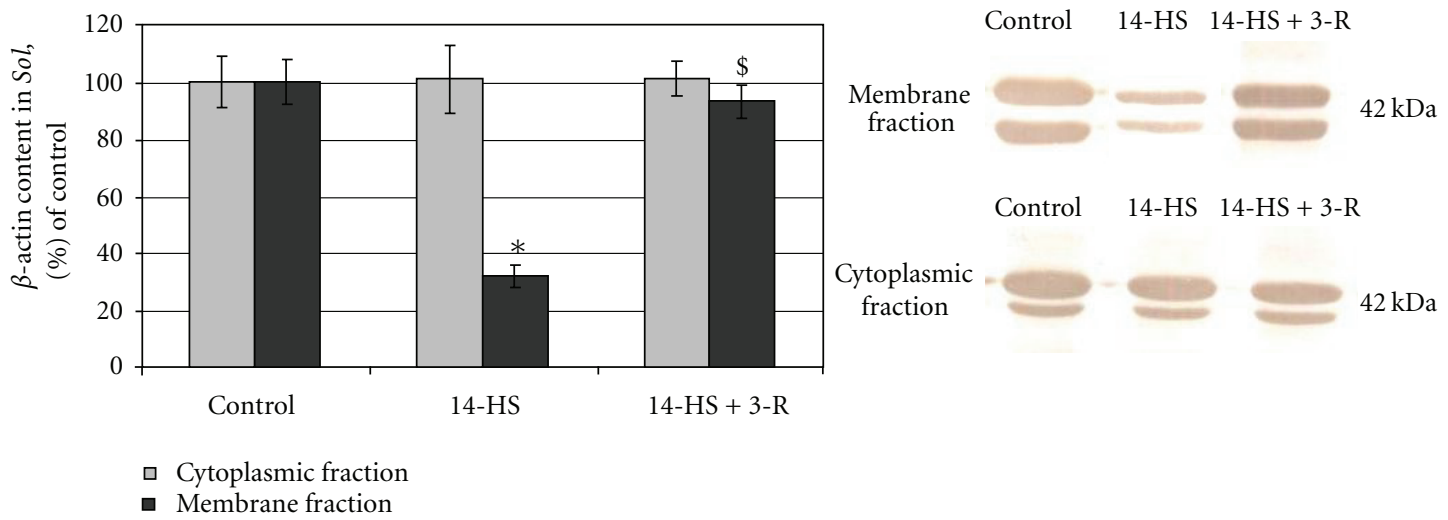

(a)
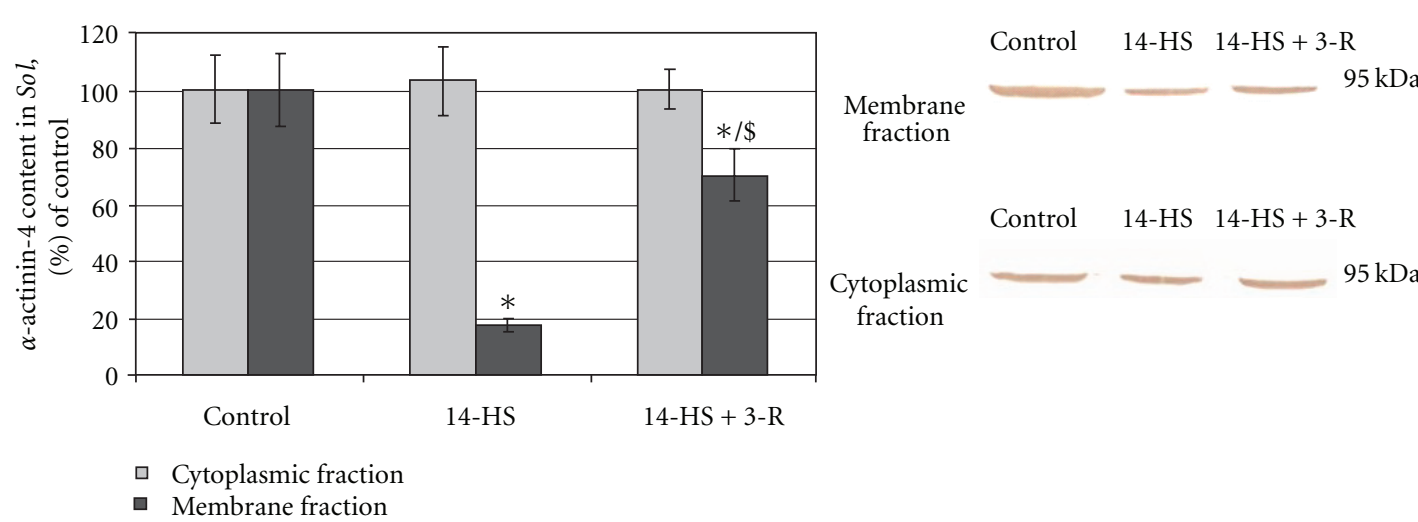

$\square$ Cytoplasmic fraction
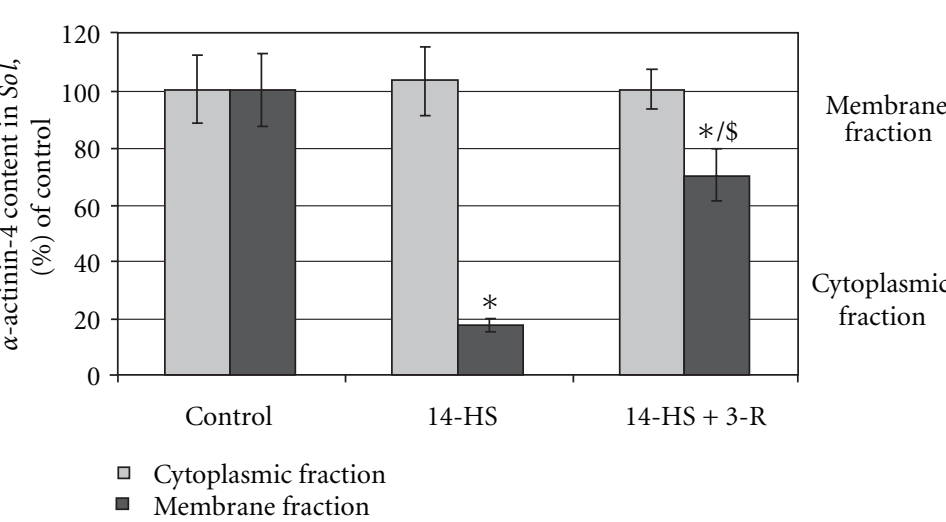

Control $\quad 14-\mathrm{HS} \quad 14-\mathrm{HS}+3-\mathrm{R}$

(b)

FIGURE 2: Protein content in the rat's Sol after 14-day gravitational unloading and 3-day reloading; (a) beta-actin, (b) alpha-actinin-4; ${ }^{*} P<0.05$ as compared to the group "Control," $\$ P<0.05$ as compared to the group "14-HS."

\begin{abstract}
"14-HS + 3-R" group in the relaxed state is also reduced with respect to "Control" group.

Other trends in gravity disuse and subsequent reloading were observed in the study of permeabilized fibers (Table 1). Transversal stiffness of the sarcolemma between the projections of Z-disk and M-line in an activated state in the "Control" group did not increase compared to the relaxed state. At the same time, the stiffness in the projection of Z-disk and M-lines increased with contraction activation and transition to the rigor. After a 14-day of antiorthostatic weighing, transversal stiffness of all sections of permeabilized fibers was reduced, but the increase of this parameter in the membrane projections of Z-disk and M-line in activated and rigor state remained. After a 3-day reloading, the sarcolemma stiffness was not different from the values in similar measurement points and conditions in the "Control" group.
\end{abstract}

3.2. Protein Content. The content of beta-actin (Figure 2(a)) in cytoplasmic protein fractions did not change after the 14day gravity disuse and subsequent three-day reloading. In the "14-HS" group, there was a reduction of beta-actin in the membrane fraction by $68 \%$ and reloading to the level of control in the "14-HS + 3-R" group.
The content of alpha-actinin-4 (Figure 2(b)), as well as beta-actin, in the cytoplasmic fraction was not changed. In the membrane fraction in the "14-HS" group, there was a decrease of alpha-actinin- 4 by $82 \%$ compared with the "Control" group, and in the "14-HS + 3-R" group, its content significantly increased compared with the group "14-HS," but did not reach the level of control and was reduced by $30 \%$.

\section{Discussion}

Previously, it was shown that the transversal stiffness of various sections of muscle fibers during the gravity disuse varies in different ways [23]. Thus, the transversal stiffness of various sections of the sarcolemma has been reducing through the day of gravity disuse, while changes in the contractile apparatus are observed after three days. The results, obtained in this paper, indicate that the reloading of the transversal stiffness also occurs in many ways.

After 14 days of hind-limbs suspension, the transversal stiffness of various sections of the sarcolemma and contractile apparatus is significantly reduced, apparently due to disassembly of the cortical cytoskeleton and calcium-dependent proteolysis of the contractile apparatus proteins, 
TABle 1: Transversal stiffness ( $\mathrm{pN} / \mathrm{nm})$ of isolated permeabilized Sol muscle fibers in liquid in relaxed, calcium-activated $(\mathrm{pCa}=4.2)$, and rigor states under 14-day gravitational unloading and 3-day reloading.

\begin{tabular}{|c|c|c|c|}
\hline State group & Relaxed & Activated $(\mathrm{pCa}=4.2)$ & Rigor \\
\hline \multicolumn{4}{|c|}{ Transversal stiffness of sarcolemma between the M-band and Z-disk projections $k_{\perp s}$} \\
\hline Control & $2.94 \pm 0.14$ & $3.09 \pm 0.17$ & $3.72 \pm 0.15 \% / \#$ \\
\hline 14-HS & $1.11 \pm 0.06^{*}$ & $1.28 \pm 0.09^{*}$ & $1.42 \pm 0.11^{* / \%}$ \\
\hline $14-\mathrm{HS}+3-\mathrm{R}$ & $2.92 \pm 0.10^{\$}$ & $2.82 \pm 0.19^{\$}$ & $3.13 \pm 0.12^{* / \$}$ \\
\hline \multicolumn{4}{|c|}{ Transversal stiffness of sarcolemma at the M-band projection $k_{\perp M l s}$} \\
\hline Control & $2.15 \pm 0.14$ & $2.73 \pm 0.13^{\%}$ & $3.25 \pm 0.19 \% / \#$ \\
\hline 14-HS & $0.99 \pm 0.11^{*}$ & $1.36 \pm 0.12^{* / \%}$ & $1.81 \pm 0.14^{* / \% / \#}$ \\
\hline $14-\mathrm{HS}+3-\mathrm{R}$ & $2.11 \pm 0.12^{\$}$ & $2.76 \pm 0.22^{\$ / \%}$ & $3.36 \pm 0.21^{\$ / \% / \#}$ \\
\hline \multicolumn{4}{|c|}{ Transversal stiffness of sarcolemma at the Z-disk projection $k_{\perp Z d s}$} \\
\hline Control & $3.25 \pm 0.18$ & $3.58 \pm 0.16^{\%}$ & $4.15 \pm 0.17 \% / \#$ \\
\hline 14-HS & $1.32 \pm 0.12^{*}$ & $1.83 \pm 0.13^{* / \%}$ & $2.01 \pm 0.12^{* / \% / \#}$ \\
\hline $14-\mathrm{HS}+3-\mathrm{R}$ & $3.01 \pm 0.13^{\$}$ & $3.33 \pm 0.11^{\$ / \%}$ & $3.96 \pm 0.17^{\$ / \% / \#}$ \\
\hline
\end{tabular}

which was discussed earlier [22, 23]. Decrease of stiffness Zdisk is especially pronounced, as shown directly by Akiyama et al. [31], may be due to the action of calpain. In addition, damages to the sarcomere, typical for type I fibers, as shown for the first time by Riley et al. [15], may also lead to a decrease in stiffness in semisarcomere area and reduced transversal stiffness. At the same time, in conditions of gravitational unloading, an increase in the proportion of type II fibers is observed [3]. It seems that gravitational unloading caused different changes in transversal stiffness of type I and type II muscle fibers. In the study of m. soleus, which predominantly consists of slow fibers, we observed the decrease of the transversal stiffness of contractile apparatus under conditions of hindlimb suspension, possibly due to sarcomere damage [15]. At the same time, the hindlimb suspension led to increase of the contractile apparatus's transversal stiffness of tibialis anterior fibers, among which predominate fast fibers [23]. Different responses of the transversal stiffness of the contractile apparatus of type I and type II fibers on gravity disuse could be dependent on the level of EMG activity. However, the performance of singlefiber electrophoresis, which is necessary for determination of fiber phenotype, connects with experimental problems due to insufficient dimension of fiber fragments used for stiffness measurements.

Stiffness of the contractile apparatus after 3-day reloading, though significantly increased in comparison with values immediately after the unloading, does not reach the level of control. Riley et al. $[13,17]$ showed that the contractile activity in the early period of readaptation (after 4.5 hours) is perceived by an atrophied muscle as eccentric load, which leads to a marked destruction of the structure of the sarcomere. At the same time after 3-day reloading after 10-day unloading of the rats, the levels of phosphorylated PKB/Akt and p70S6K significantly increased relative to control, which may be indicative of an intensification of protein synthesis [18]. Taking these data into account, we can assume that in the first few hours after unloading, there is an even greater reduction of transversal stiffness of the contractile apparatus compared to the state immediately after unloading. Next, the intensification of protein synthesis leads to the repair of the sarcomere structure, but after 3 days of readaptation, this process is not completed, and that is reflected in the partial recovery of the values of the transversal stiffness of contractile apparatus.

At the same time, the transversal stiffness of different parts of the sarcolemma does not differ from the control level after 3 days of reloading after a 14-day antiorthostatic suspension. At the same time in the early stages of gravitational unloading, reduced transversal stiffness of the sarcolemma was seen in a day after gravity disuse, while changes in stiffness of the contractile apparatus took place just in three days [23]. Therefore, we can assume that change in the transversal stiffness of sarcolemma is more sensitive to changes in external mechanical conditions, which is of interest in connection with the ongoing search for mechanosensitive cell structures.

By analogy with the results of Costa et al. [32], obtained on human aorta cells, we can assume that changes in transversal stiffness of permeabilized fibers are associated with changes in the submembrane actin cytoskeleton, which is consistent with the data of Collinsworth et al. [33]. Reduction of the transversal stiffness of permeabilized fibers can be connected with the destruction of filaments formed by nonsarcomeric actin (beta-actin) and changes in the content of the actin-binding proteins, including alpha-actinin-4.

To test this hypothesis, we have analyzed the relative content of beta-actin in the cytoplasmic and membrane fraction of the total protein. The data indicate that the content of beta-actin in the cytoplasmic fraction did not change either as a result of a 14-day unloading, or in the subsequent threeday reloading. However, the content of beta-actin protein in the membrane fraction after disuse was reduced by more than 3 times compared to the control, but after 3 days of reloading did not differ from control, which correlated with changes in transversal stiffness of the sarcolemma. 
The destruction of the cortical cytoskeleton naturally leads to a transient increase in the content of G-actin, which in its turn can trigger several signaling pathways, such as SRF dependent [34].

However, the cause of the destruction of the cortical cytoskeleton can be dissociation from the actin of actin-binding proteins, including alpha-actinin-4. Our data suggest that the content of alpha-actinin- 4 in the protein cytoplasmic fraction does not change. The dynamics of change of the content of alpha-actinin-4 in the membrane fraction is similar to that for beta-actin although full reloading of its relative content after 3-day readaptation does not occur. Alpha-actinin-4 is a nonmuscle isoform of alpha-actinin, a protein belonging to the family of spectrin [35]. It functions as an antiparallel homodimer, binding the ends of actin filaments with each other [36]. In addition, alpha-actinin4 connects the actin cytoskeleton to the membrane, and cortical cytoskeleton interacts with the cytoplasmic signaling proteins [37]. Alpha-actinin-4 can bind calcium ions at micromolar concentrations, and calcium concentration greater than $10^{-7} \mathrm{M}$ completely inhibits its binding to actin [38], as well as tyrosine phosphorylation of focal adhesive kinase within actin-binding domain [39]. We have previously shown that within a one day of gravitational unloading, the basal content of calcium ions increases in the fibers of soleus muscle of a rat [22]. In addition, we know that already by the 2nd day of gravitational unloading, accumulation of calcium ions in the fibers of mouse soleus muscle occurs [24, 25].

\section{Conclusion}

We can assume that as a result of gravitational unloading due to changes in tension, there is deformation of the cortical cytoskeleton, which leads to the dissociation of alphaactinin-4 from subsarcolemmal actin and, consequently, to the cortical cytoskeleton destruction and reduction of the stiffness of the membrane. However, on the other hand, changes in membrane tension can modulate ion channels work and lead to an accumulation of calcium ions. The increase of calcium ions may contribute to the binding of alpha-actinin-4, the dissociation of the latter from cortical actin, and subsequent degradation of submembrane cytoskeleton. And in fact, in both cases, the accumulation of calcium and G-actin as a result of the cortical cytoskeleton degradation will lead to the launch of a number of signaling pathways, proteolysis ones in the first place, and the formation of adaptive response to microgravity conditions.

\section{Conflict of Interests}

No conflict of interests is declared by the author.

\section{Acknowledgments}

The author expresses gratitude to V. A. Kurushin, E. V. Ponomareva, and E. G. Altaeva for their assistance in carrying out the experiments. The financial support of the Russian Foundation for Basic Research (RFBR Grant no. 10-04-00 106-a) and program of fundamental research of SSC RFIBMP RAS is greatly acknowledged.

\section{References}

[1] F. W. Booth and J. R. Kelso, "Effect of hind limb immobilization on contractile and histochemical properties of skeletal muscle," Pflugers Archiv European Journal of Physiology, vol. 342, no. 3, pp. 231-238, 1973.

[2] D. Desplanches, M. H. Mayet, E. I. Ilyina-Kakueva, B. Sempore, and R. Flandrois, "Skeletal muscle adaptation in rats flown on Cosmos 1667," Journal of Applied Physiology, vol. 68, no. 1, pp. 48-52, 1990.

[3] V. J. Caiozzo, F. Haddad, M. J. Baker, R. E. Herrick, N. Prietto, and K. M. Baldwin, "Microgravity-induced transformations of myosin isoforms and contractile properties of skeletal muscle," Journal of Applied Physiology, vol. 81, no. 1, pp. 123-132, 1996.

[4] R. R. Roy, H. Zhong, S. C. Bodine et al., "Fiber size and myosin phenotypes of selected rhesus lower limb muscles after a 14day spaceflight," Journal of Gravitational Physiology, vol. 7, no. 1, p. S45, 2000.

[5] K. C. Darr and E. Schultz, "Hindlimb suspension suppresses muscle growth and satellite cell proliferation," Journal of Applied Physiology, vol. 67, no. 5, pp. 1827-1834, 1989.

[6] R. J. Talmadge, R. R. Roy, and V. R. Edgerton, "Distribution of myosin heavy chain isoforms in non-weight-bearing rat soleus muscle fibers," Journal of Applied Physiology, vol. 81, no. 6, pp. 2540-2546, 1996.

[7] D. Pette and R. S. Staron, "Transitions of muscle fiber phenotypic profiles," Histochemistry and Cell Biology, vol. 115, no. 5, pp. 359-372, 2001.

[8] R. J. Talmadge, R. R. Roy, V. J. Caiozzo, and V. Reggie Edgerton, "Mechanical properties of rat soleus after long-term spinal cord transection," Journal of Applied Physiology, vol. 93, no. 4, pp. 1487-1497, 2002.

[9] G. R. Adams, V. J. Caiozzo, and K. M. Baldwin, "Skeletal muscle unweighting: spaceflight and ground-based models," Journal of Applied Physiology, vol. 95, no. 6, pp. 2185-2201, 2003.

[10] J. J. Widrick, G. F. Maddalozzo, H. Hu, J. C. Herron, U. T. Iwaniec, and R. T. Turner, "Detrimental effects of reloading recovery on force, shortening velocity, and power of soleus muscles from hindlimb-unloaded rats," American Journal of Physiology, Regulatory Integrative and Comparative Physiology, vol. 295, no. 5, pp. R1585-R1592, 2008.

[11] K. Lee, Y. S. Lee, M. Lee, M. Yamashita, and I. Choi, "Mechanics and fatigability of the rat soleus muscle during early reloading," Yonsei Medical Journal, vol. 45, no. 4, pp. 690702, 2004.

[12] K. S. McDonald and R. H. Fitts, "Effect of hindlimb unloading on rat soleus fiber force, stiffness, and calcium sensitivity," Journal of Applied Physiology, vol. 79, no. 5, pp. 1796-1802, 1995.

[13] D. A. Riley, S. Ellis, G. R. Slocum et al., "In-flight and postflight changes in skeletal muscles of SLS-1 and SLS-2 spaceflown rats," Journal of Applied Physiology, vol. 81, no. 1, pp. 133-144, 1996.

[14] D. A. Riley, S. Ellis, C. S. Giometti et al., "Muscle sarcomere lesions and thrombosis after spaceflight and suspension unloading," Journal of Applied Physiology, vol. 73, pp. 33S-43S, 1992.

[15] D. A. Riley, E. I. Ilyina-Kakueva, S. Ellis, J. L. W. Bain, G. R. Slocum, and F. R. Sedlak, "Skeletal muscle fiber, nerve, and 
blood vessel breakdown in space-flown rats," FASEB Journal, vol. 4, no. 1, pp. 84-91, 1990.

[16] D. A. Riley, J. L. Thompson, B. B. Krippendorf, and G. R. Slocum, "Review of spaceflight and hindlimb suspension unloading induced sarcomere damage and repair," Basic and Applied Myology, vol. 5, no. 2, pp. 139-145, 1995.

[17] D. A. Riley, J. L. W. Bain, J. L. Thompson et al., "Decreased thin filament density and length in human atrophic soleus muscle fibers after spaceflight," Journal of Applied Physiology, vol. 88, no. 2, pp. 567-572, 2000.

[18] T. Sugiura, N. Abe, M. Nagano et al., "Changes in PKB/Akt and calcineurin signaling during recovery in atrophied soleus muscle induced by unloading," American Journal of Physiology, Regulatory Integrative and Comparative Physiology, vol. 288, no. 5, pp. R1273-R1278, 2005.

[19] X. J. Musacchia, J. M. Steffen, R. D. Fell, and M. J. Dombrowski, "Skeletal muscle response to spaceflight, whole body suspension, and recovery in rats," Journal of Applied Physiology, vol. 69, no. 6, pp. 2248-2253, 1990.

[20] K. S. Litvinova, P. P. Tarakin, N. M. Fokina, V. E. Istomina, I. M. Larina, and B. S. Shenkman, "Reloading of rat soleus after hindlimb unloading and serum insulin-like growth factor 1," Ross Fiziol Zh Im I M Sechenova, vol. 93, no. 10, pp. 1143-1155, 2007.

[21] R. J. Bloch and H. Gonzalez-Serratos, "Lateral force transmission across costameres in skeletal muscle," Exercise and Sport Sciences Reviews, vol. 31, no. 2, pp. 73-78, 2003.

[22] I. V. Ogneva, V. A. Kurushin, E. G. Altaeva, E. V. Ponomareva, and B. S. Shenkman, "Effect of short-term gravitational unloading on rat and mongolian gerbil muscles," Journal of Muscle Research and Cell Motility, vol. 30, no. 7-8, pp. 261$265,2009$.

[23] I. V. Ogneva, "Transversal stiffness of fibers and desmin content in leg muscles of rats under gravitational unloading of various durations," Journal of Applied Physiology, vol. 109, no. 6, pp. 1702-1709, 2010.

[24] C. P. Ingalls, G. L. Warren, and R. B. Armstrong, "Intracellular $\mathrm{Ca} 2+$ transients in mouse soleus muscle after hindlimb unloading and reloading," Journal of Applied Physiology, vol. 87, no. 1, pp. 386-390, 1999.

[25] C. P. Ingalls, J. C. Wenke, and R. B. Armstrong, "Time course changes in $[\mathrm{Ca} 2+] \mathrm{i}$, force, and protein content in hind-limbsuspended mouse soleus muscles," Aviation Space and Environmental Medicine, vol. 72, no. 5, pp. 471-476, 2001.

[26] I. V. Ogneva, D. V. Lebedev, and B. S. Shenkman, “Transversal stiffness and young's modulus of single fibers from rat soleus muscle probed by atomic force microscopy," Biophysical Journal, vol. 98, no. 3, pp. 418-424, 2010.

[27] E. Morey-Holton, R. K. Globus, A. Kaplansky, and G. Durnova, "The hindlimb unloading rat model: literature overview, technique update and comparison with space flight Data," Advances in Space Biology and Medicine, vol. 10, pp. $7-$ 40, 2005.

[28] L. Stevens, Y. Mounier, and X. Holy, "Functional adaptation of different rat skeletal muscles to weightlessness," American Journal of Physiology, vol. 264, no. 4, pp. R770-R776, 1993.

[29] R. Vitorino, R. Ferreira, M. Neuparth et al., "Subcellular proteomics of mice gastrocnemius and soleus muscles," Analytical Biochemistry, vol. 366, no. 2, pp. 156-169, 2007.

[30] H. Towbin, T. Staehelin, and J. Gordon, "Electrophoretic transfer of proteins from polyacrylamide gels to nitrocellulose sheets: procedure and some applications," Proceedings of the National Academy of Sciences of the United States of America, vol. 76, no. 9, pp. 4350-4354, 1970.
[31] N. Akiyama, Y. Ohnuki, Y. Kunioka, Y. Saeki, and T. Yamada, "Transverse stiffness of myofibrils of skeletal and cardiac muscles studied by atomic force microscopy," Journal of Physiological Sciences, vol. 56, no. 2, pp. 145-151, 2006.

[32] K. D. Costa, A. J. Sim, and F. C. P. Yin, "Non-Hertzian approach to analyzing mechanical properties of endothelial cells probed by atomic force microscopy," Journal of Biomechanical Engineering, vol. 128, no. 2, pp. 176-184, 2006.

[33] A. M. Collinsworth, S. Zhang, W. E. Kraus, and G. A. Trus-key, "Apparent elastic modulus and hysteresis of skeletal muscle cells throughout differentiation," American Journal of Physiology, vol. 283, no. 4, pp. 1219-1227, 2002.

[34] K. Kuwahara, T. Barrientos, G. C. T. Pipes, S. Li, and E. N. Olson, "Muscle-specific signaling mechanism that links actin dynamics to serum response factor," Molecular and Cellular Biology, vol. 25, no. 8, pp. 3173-3181, 2005.

[35] M. J. F. Broderick and S. J. Winder, "Towards a complete atomic structure of spectrin family proteins," Journal of Structural Biology, vol. 137, no. 1-2, pp. 184-193, 2002.

[36] H. Youssoufian, M. McAfee, and D. J. Kwiatkowski, "Cloning and chromosomal localization of the human cytoskeletal $\alpha$ actinin gene reveals linkage to the $\beta$-spectrin gene," American Journal of Human Genetics, vol. 47, no. 1, pp. 62-72, 1990.

[37] M. D. Baron, M. D. Davison, P. Jones, and D. R. Critchley, "The structure and function of alpha-actinin," Biochemical Society Transactions, vol. 15, no. 5, pp. 796-798, 1987.

[38] T. Parr, G. T. Waites, B. Patel, D. B. Millake, and D. R. Critchley, "A chick skeletal-muscle $\alpha$-actinin gene gives rise to two alternatively spliced isoforms which differ in the EF-hand Ca2+-binding domain," European Journal of Biochemistry, vol. 210, no. 3, pp. 801-809, 1992.

[39] G. T. Waites, I. R. Graham, P. Jackson et al., "Mutually exclusive splicing of calcium-binding domain exons in chick $\alpha$ - actinin," Journal of Biological Chemistry, vol. 267, no. 9, pp. 6263-6271, 1992. 

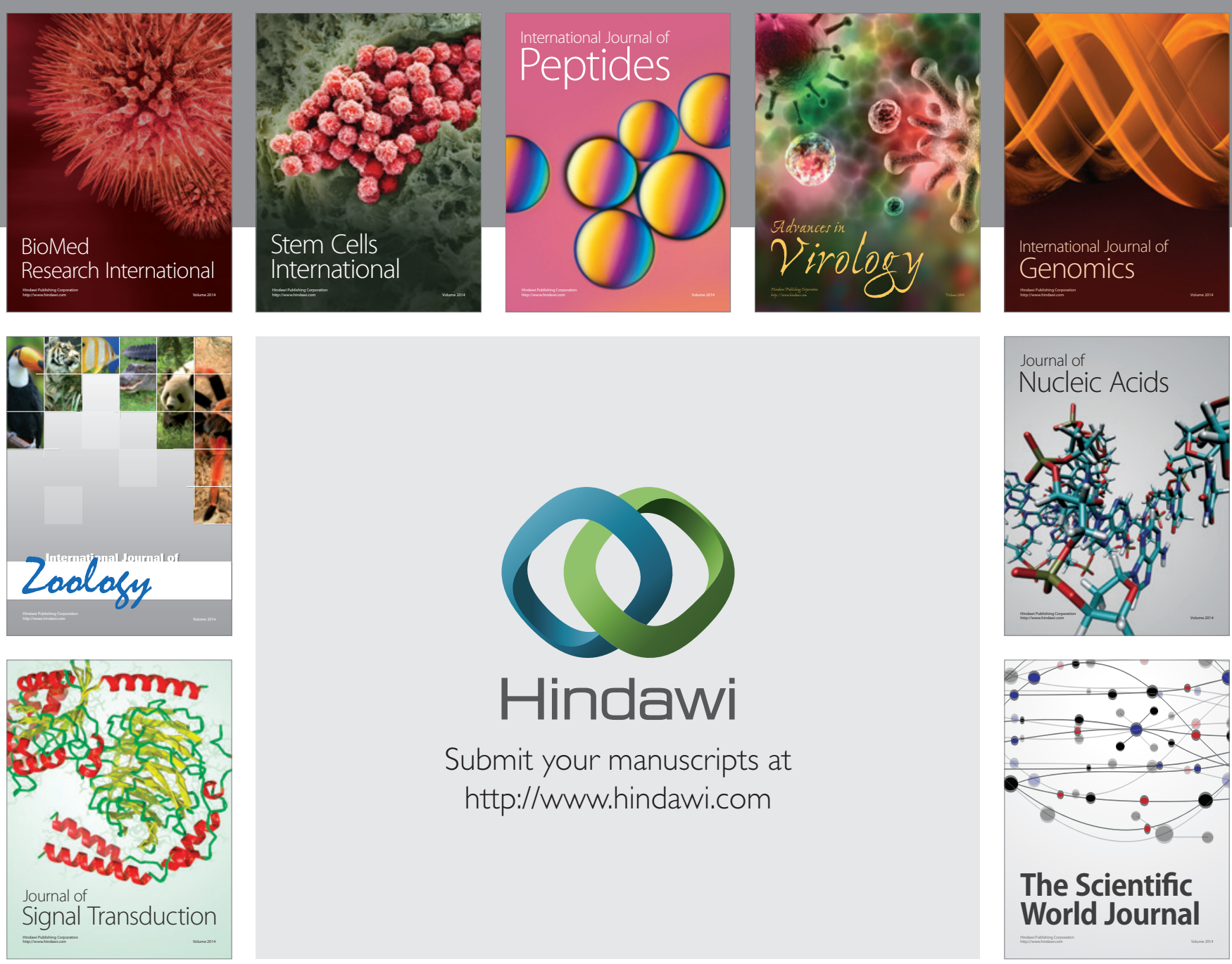

Submit your manuscripts at

http://www.hindawi.com
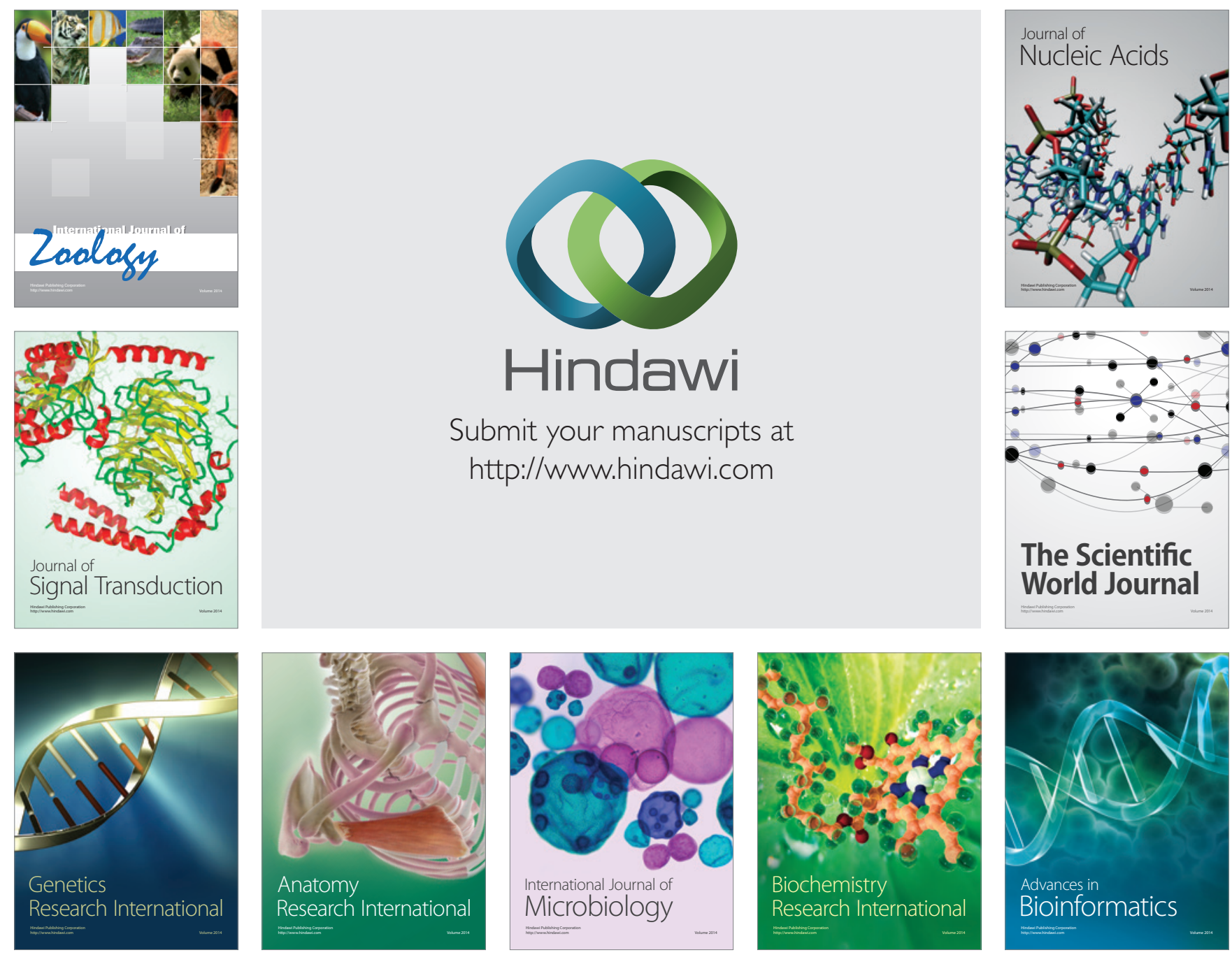

The Scientific World Journal
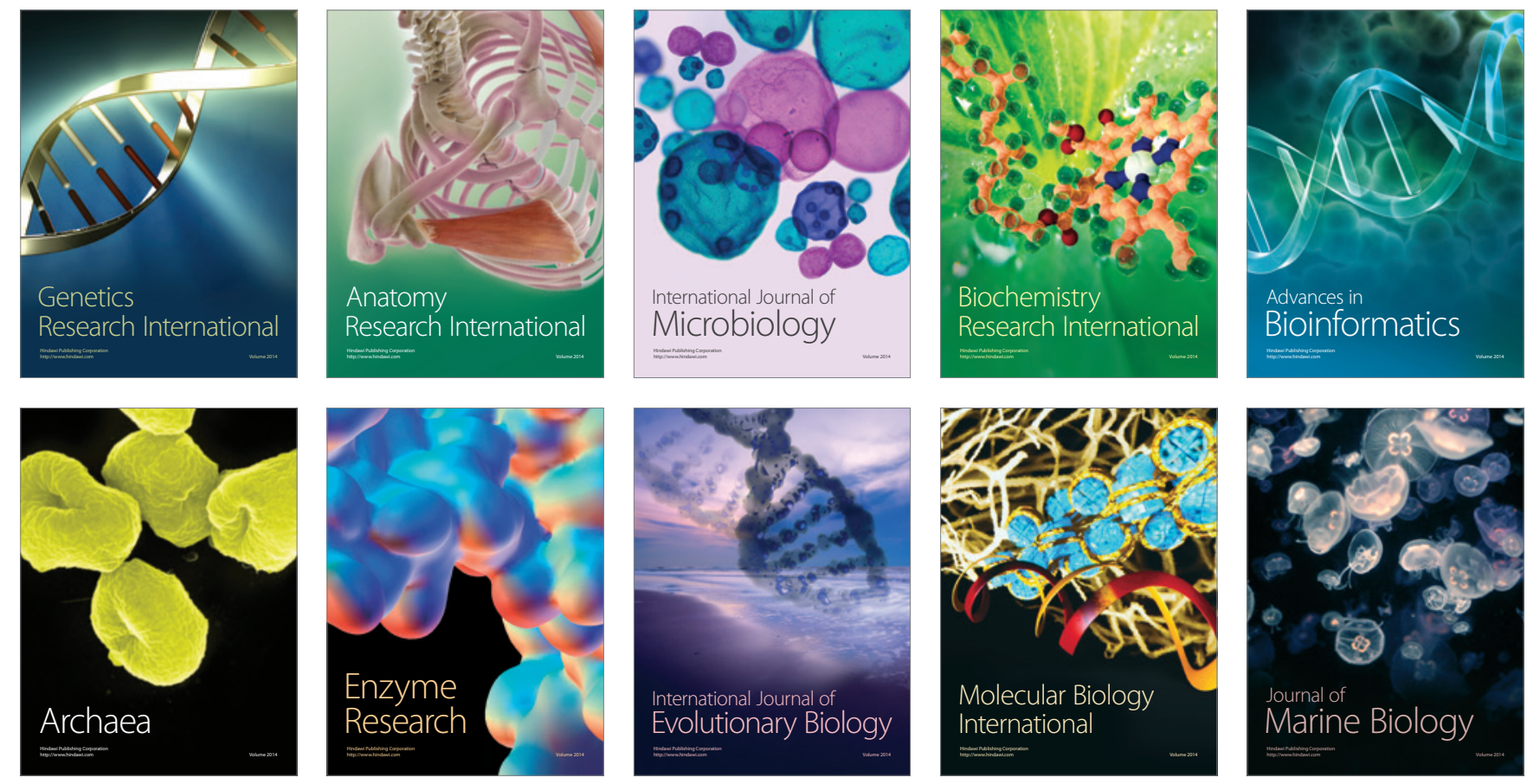